\title{
Initial experience with a multiple parallel guidewire support system for complex tortuous aortic arch navigation and great vessel catheterization: technical note
}

\author{
Leonardo Rangel-Castilla, MD, 1,2 Hakeem J. Shakir, MD,,2 and Adnan H. Siddiqui, MD, PhD'-5 \\ Departments of ${ }^{1}$ Neurosurgery and ${ }^{3}$ Radiology, Jacobs School of Medicine and Biomedical Sciences, University at Buffalo, \\ State University of New York; ${ }^{2}$ Department of Neurosurgery, Gates Vascular Institute, Kaleida Health; ${ }^{4}$ Toshiba Stroke and \\ Vascular Research Center, University at Buffalo, State University of New York; and ${ }^{5}$ Jacobs Institute, Buffalo, New York
}

\begin{abstract}
The ability to traverse an anatomically challenging and complex arch is paramount to the success of any neuroendovascular procedure. With age, the aortic arch becomes elongated, calcified, and less compliant. The authors present the initial experience with a multiple parallel guidewire system (ZigiWire Mode 3) for catheterization through a complex tortuous aortic arch to access extracranial vessels. The ZigiWire is an organized guidewire system that uses consecutive delivery of 3 small-diameter (0.014-inch) guidewires that are progressively advanced in parallel to secure support-wire access. The authors have found it useful in situations in which traditional methods for great-vessel access have failed. Moreover, the progressive construction of a large wire from smaller wires prevents "kickback" force from a single larger guidewire, allowing stable distal access. The authors have been able to advance different diagnostic and guide catheters over the ZigiWire. This guidewire has allowed them to successfully complete neuroendovascular procedures in patients who were previously considered unsuitable for the procedure because of tortuous vascular access.
\end{abstract}

https://thejns.org/doi/abs/10.3171/2017.1.FOCUS16494

KEY WORDS tortuous carotid artery; catheterization; great vessels; guidewire support

$\mathrm{A}$ CCESSIBILITY to cervical and cerebrovascular structures is essential for the performance of neuroendovascular procedures. The neurointerventionist must be able to navigate the aortic arch and obtain access to the supra-aortic vessels. The ability to traverse an anatomically challenging and complex arch is paramount to the success of any neuroendovascular procedure. With age, the aortic arch becomes elongated, calcified, and less compliant, and the takeoff of the great vessels becomes more angulated..$^{1-4}$ At times, catheterization of the great vessels is not possible, and direct cutdown for direct access is necessary..$^{1-4}$ We describe the initial experience with a multiple parallel guidewire system (ZigiWire Mode 3, Vadiswire Corp.) for catheterization of complex, tortuous extracranial vessels during neuroendovascular procedures.

\section{Presentation of Cases}

We present 6 patients with complex tortuous aortic arches and great vessels, each of whom required a neuroendovascular procedure. Initial catheterization of the great vessels with standard catheters (Berenstein, Vitek, or Headhunter, Cook Medical; or Simmons 2, Terumo) and standard wires (0.035-inch or 0.038-inch Glidewire, Terumo; or Amplatz, Boston Scientific) was not successful in obtaining stable positioning of the guide catheter in supra-aortic vessels in any of them. In each case, the ZigiWire multiple parallel guidewire was used to access and catheterize the target great vessel (Table 1).

This study was approved by the University at Buffalo Health Sciences institutional review board. Informed consent was obtained.

\section{Technique}

The ZigiWire Mode 3 is an organized guidewire system that uses consecutive delivery of 3 small-diameter guidewires that are progressively advanced in parallel to 
TABLE 1. Summary of the 6 patients in whom the ZigiWire was used

\begin{tabular}{ccllll}
\hline Case No. & Age (yrs), Sex & Arch Type & \multicolumn{1}{c}{ Great Vessel Access } & Guide Catheter Used & Procedure \\
\hline 1 & $63, \mathrm{~F}$ & III & Right CCA & Neuron Max & Mechanical thrombectomy \\
\hline 2 & $71, \mathrm{~F}$ & III/bovine & Left CCA & Neuron Max & Mechanical thrombectomy \\
\hline 3 & $74, \mathrm{M}$ & III & Left CCA & Neuron Max & Mechanical thrombectomy \\
\hline 4 & $72, \mathrm{~F}$ & III/bovine & Left CCA & Cook-Shuttle & CAS \\
\hline 5 & $63, \mathrm{M}$ & III/bovine & Left CCA \& ECA & Cook-Shuttle & AVF embolization \\
\hline 6 & $75, \mathrm{~F}$ & III & Brachiocephalic trunk, right CCA & Mo.Ma & Attempted ICA revascularization \\
\hline
\end{tabular}

$\mathrm{AVF}=$ arteriovenous fistula; $\mathrm{CAS}=$ carotid artery stenting; $\mathrm{CCA}=$ common carotid artery; ECA = external carotid artery; ICA = internal carotid artery.

secure support wire access (Fig. 1). The diameter of each microguidewire is 0.014 inch $(0.36 \mathrm{~mm})$.

With the patient under conscious sedation and local anesthesia, a femoral sheath (6-9 Fr) was introduced into the right femoral artery. After multiple failed attempts to catheterize the great vessels using traditional diagnostic catheters and wires, the ZigiWire Mode 3 was delivered through a diagnostic catheter (Simmons 2, Terumo; or Vitek, Cook Medical), and the construct was advanced into the aortic arch. Once the takeoff of the great vessel of interest was engaged with the diagnostic catheter, each of the 0.014-inch microguidewires of the ZigiWire was advanced individually, distally into the common carotid artery. After all 3 guidewires were in place, the diagnostic catheter was advanced into the common carotid artery. The ZigiWire was exchanged for a 0.035 - or 0.038 -inch exchange length Glidewire. The diagnostic catheter was then exchanged for a guide catheter. Another alternative was to introduce the ZigiWire through an intermediate catheter (e.g., Vitek, Cook Medical) and through the desired guide/shuttle catheter. The intermediate catheter is engaged into the takeoff of the great vessel, each of the 0.014-inch microguidewires of the ZigiWire was advanced individually, distally, into the common carotid artery. After all 3 guidewires were in place, the guide/shuttle catheter was advanced into the common carotid artery.

\section{Results}

During a 17-month period (October 1, 2014-April 31, 2016), we used the ZigiWire guidewire through a transfemoral approach in 6 patients. Planned procedures included 3 intracranial mechanical thrombectomies, 1 carotid stent placement, 1 dural arteriovenous fistula embolization, and 1 attempted internal carotid artery revascularization (Table 1). We gained access to the target vessels (brachiocephalic trunk, right common carotid artery, and left common carotid artery) in all 6 cases. Two illustrative cases are presented in Figs. 2 and 3. There were no complications associated with the use of this novel guidewire.

\section{Discussion}

As people age, the aortic arch becomes calcified, elongated, and less compliant. The primary driver for this is believed to be chronic hypertension. In younger patients, the origin of the great supra-aortic vessels is at the apex of the aortic arch. As the arch elongates, it preferentially extends beyond the origin of the left subclavian artery, which, along with other supra-aortic great vessels, tethers the aortic ostial segment. This necessarily results in much greater difficulty in catheterization of these great vessels from a transfemoral approach. Typically, this situation is

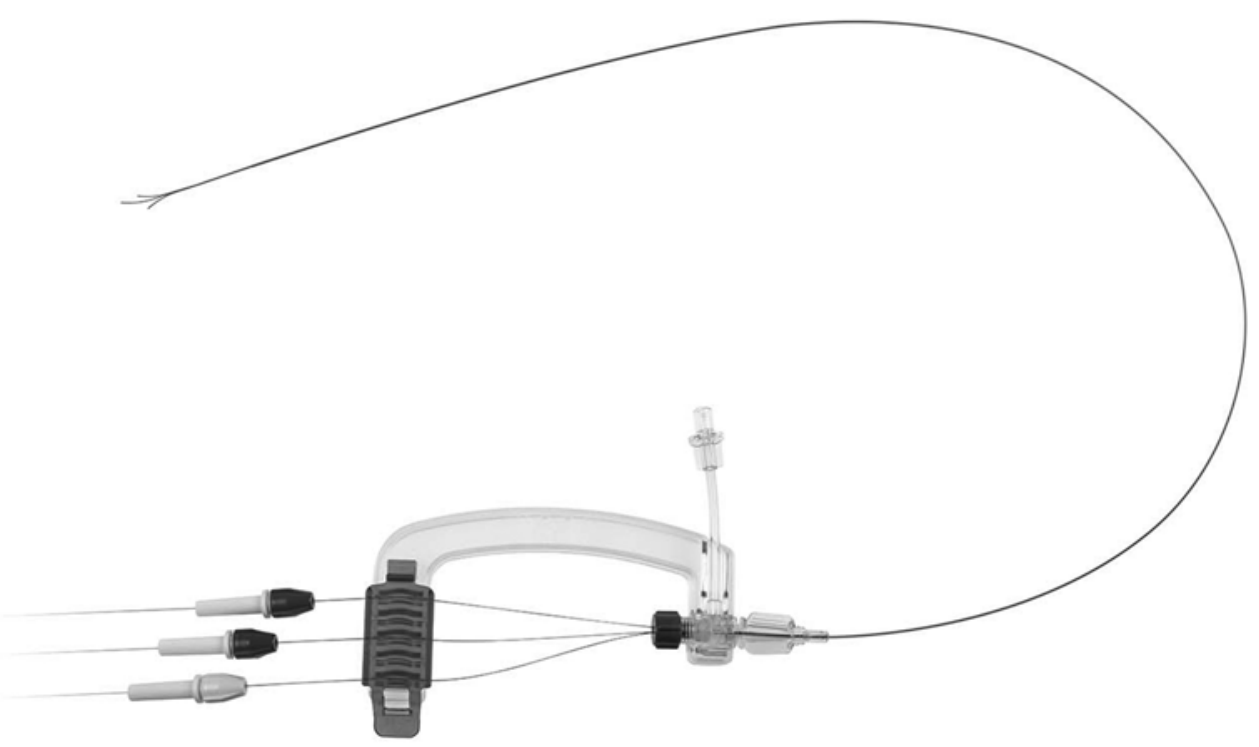

FIG. 1. ZigiWire Mode 3. Illustration of this multiple parallel guidewire system. Published with permission from Vadiswire Corp. 

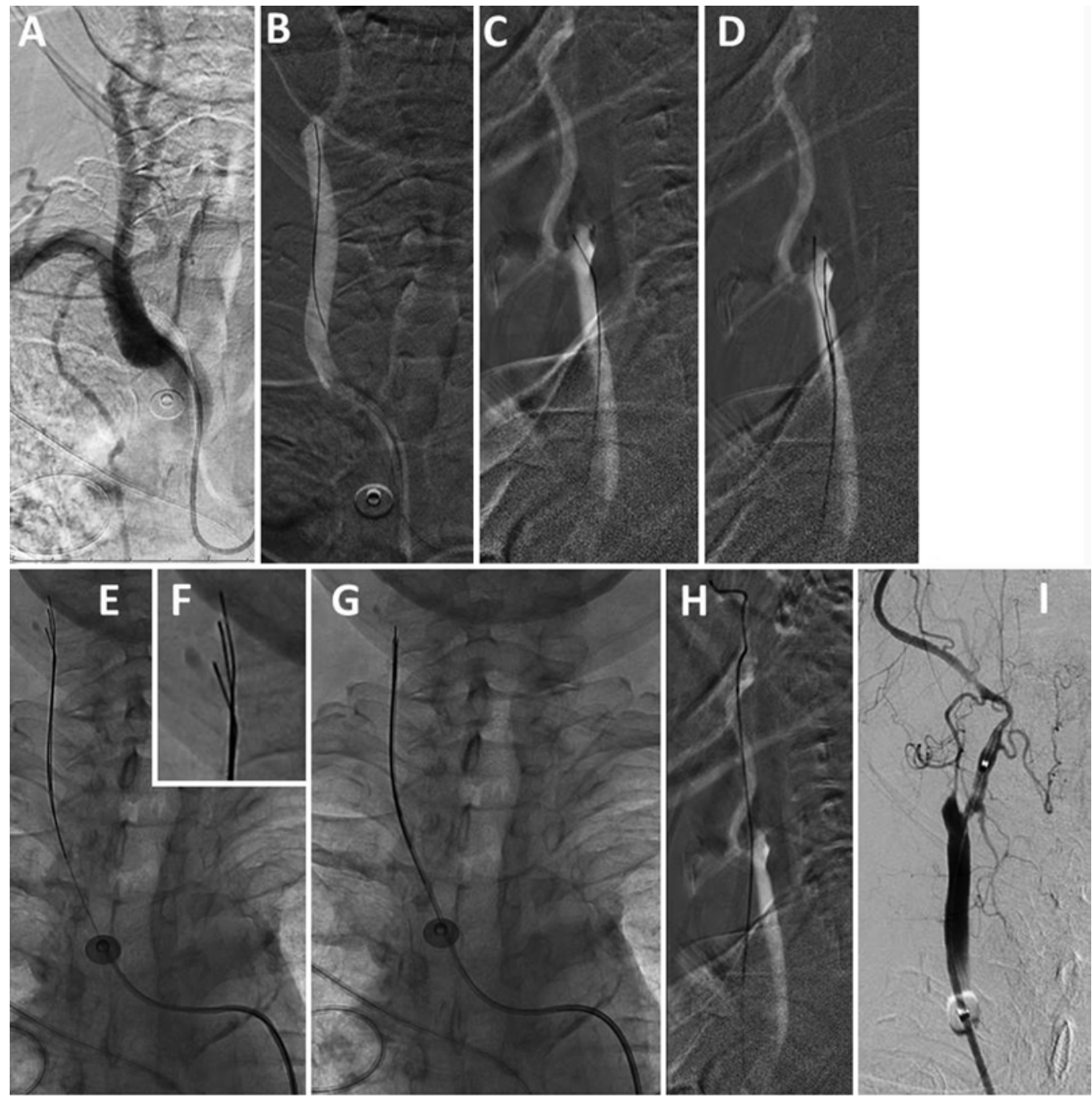

FIG. 2. Case 6. Use of the ZigiWire for tortuous extracranial vessel access. A: Brachiocephalic trunk injection with a Simmons 2 diagnostic catheter. B and $\mathrm{C}$ : Anteroposterior $(\mathrm{B})$ and lateral $(\mathrm{C})$ views under roadmap guidance. The first of the 3 consecutive wires is advanced into the right common carotid artery. D: The second wire is advanced. $\mathrm{E}$ and $\mathrm{F}$ : The third wire is advanced (panel $\mathrm{F}$ is a close-up image of $\mathrm{E}$ ). G: Once stable support is obtained, the Simmons 2 catheter is advanced into the right common carotid artery. H: The ZigiWire is exchanged for a 0.038 -inch stiff Glidewire. I: The Simmons 2 diagnostic catheter is then exchanged for a Mo.Ma balloon guide catheter.

adequately addressed by using recurve catheters, such as a Vitek or Simmons 2 catheter. However, in a small number of cases, the tortuosity of the arch and further elongation and tortuosity of the great vessels themselves are such that any attempts with standard glide wires through these recurve catheters result in a catheter "kickback" into the aorta and failure to achieve distal access.

The difficult arch is best visualized on aortic arch angiograms or high-quality $\mathrm{CT}$ and/or MR angiograms. Classification of the elongation of the aortic arch (Types I-III) correlates with increasing difficulty in great vessel cannulation and an increased risk of complications when using femoral artery access. ${ }^{1-4}$ In addition to tortuosity, these vessels are also commonly atherosclerotic and calcified and therefore not compliant. Moreover, positive forces on guidewires to gain distal access translate into pushback or retrograde movement of guiding catheters and herniation into the arch. Anatomical variations in the aortic arch itself, such as the so-called bovine arch, also complicate access to the great vessels. ${ }^{1-4}$ Access in these cases takes longer and requires greater efforts with increasingly higher risk manipulation. These arches also frequently have atherosclerotic plaque on walls and vessel ostia, posing a markedly higher risk of intraprocedural embolic complications. ${ }^{1-5}$

Excessive vascular tortuosity is responsible for failed endovascular therapy in $4 \%-6 \%$ of cases. ${ }^{1-4}$ Several techniques for overcoming difficult aortic arch and great vessel anatomy have been described. Exchanging a diagnostic catheter over a stiff exchange wire is the standard maneuver, ${ }^{5}$ but this strategy typically fails in complex Type III arches with great vessel tortuosity because of guide catheter and wire pushback and inability to access distal cervical vasculature. Transbrachial or transradial approaches are excellent for posterior circulation access but are similarly complicated during carotid artery access. Direct per- 

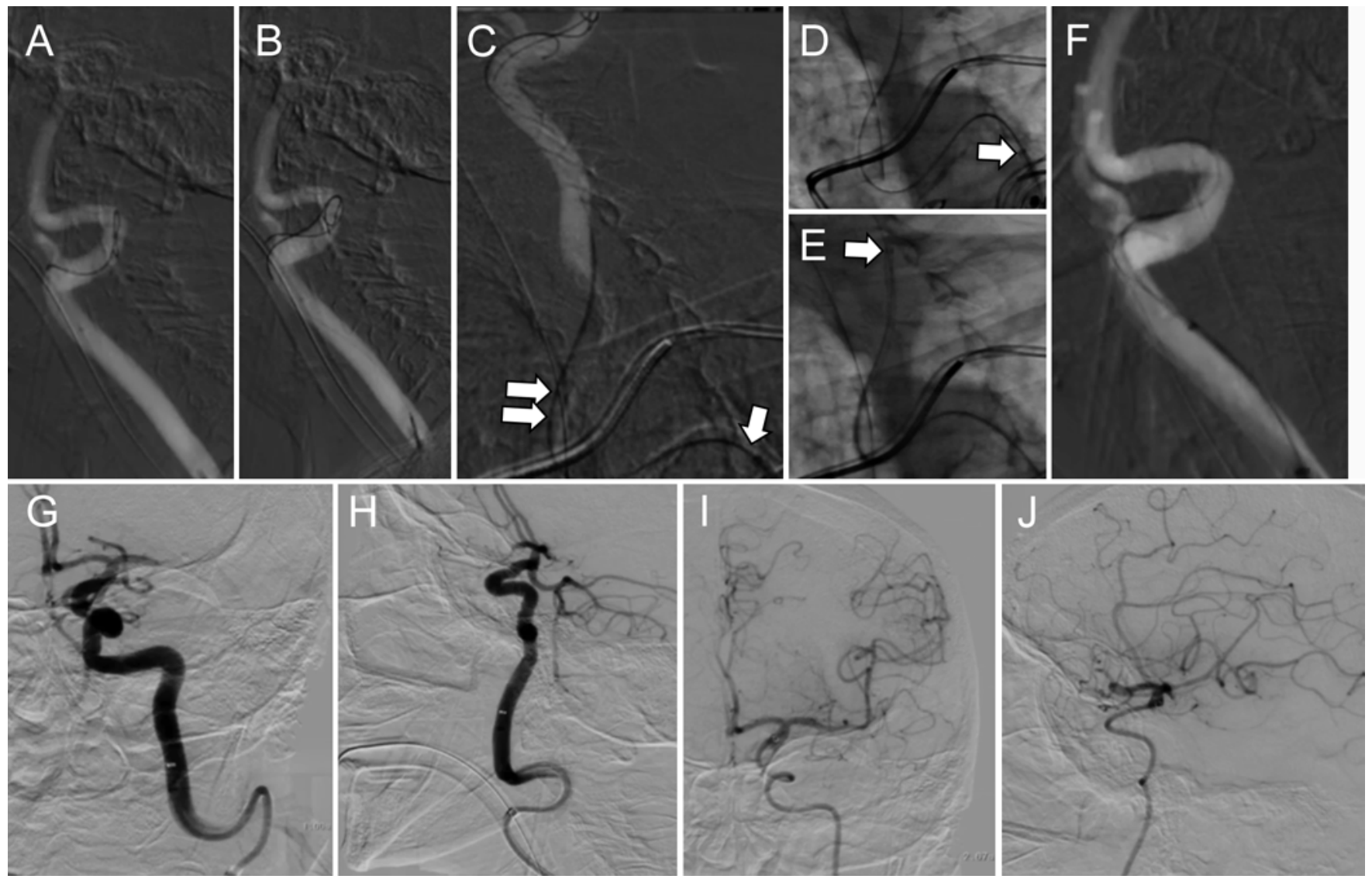

FIG. 3. Case 3. Use of the ZigiWire for tortuous extracranial vessel access. A: Lateral view under roadmap guidance. The first of the 3 consecutive wires is advanced into the left common and internal carotid arteries. B: The second wire is advanced. C: The third wire is advanced (anteroposterior view). Notice the guide (single arrow) and an intermediate catheter (double arrows). D and E: Radiographs demonstrating the guide catheter advanced from the aortic arch (arrow, D) into the common carotid artery (arrow, E) over the ZigiWire and intermediate catheter. F: Lateral view under roadmap guidance showing the reperfusion catheter climbing up over 2 of the consecutive wires. G and $\mathbf{H}$ : Intracranial views of the left internal carotid artery injection demonstrating complete occlusion of the left middle cerebral artery. I and J: Complete revascularization of the left middle cerebral artery after mechanical thrombectomy with a stent retriever.

cutaneous puncture of the carotid artery or open cutdown are other approaches, both of which increase the risk of carotid artery dissection, wound infection, and neck hematoma.

We have found the ZigiWire system useful in situations in which traditional methods for great vessel access have failed. The consecutive delivery of small wires (0.014inch) allows us to sequentially build a stable construct with a distal wire over which a diagnostic catheter or an interventional guide catheter can be advanced. The progressive construction of a large wire from smaller wires also prevents the kickback force from a single larger guidewire, allowing distal and stable access. Using the ZigiWire system, we were able to advance different catheters, including a Simmons 2 5-Fr diagnostic catheter, a 6-Fr Shuttle Guiding Sheath (Cook Medical), and a Mo.Ma 9-Fr balloon guide catheter (Medtronic) (Table 1).

\section{Conclusions}

The described guidewire system offers a method to gain vascular access in certain patients with difficult aortic arch anatomy. It has allowed us to successfully complete neuroendovascular procedures in patients previously considered unsuitable for the procedure because of tortuous vascular access.

\section{Acknowledgments}

We thank Paul H. Dressel, BFA, for preparation of the illustrations and Debra J. Zimmer for editorial assistance.

\section{References}

1. Chang FC, Tummala RP, Jahromi BS, Samuelson RM, Siddiqui AH, Levy EI, et al: Use of the 8 French Simmons-2 guide catheter for carotid artery stent placement in patients with difficult aortic arch anatomy. J Neurosurg 110:437441, 2009

2. Lam RC, Lin SC, DeRubertis B, Hynecek R, Kent KC, Faries PL: The impact of increasing age on anatomic factors affecting carotid angioplasty and stenting. J Vasc Surg 45:875880,2007 
3. Lin SC, Trocciola SM, Rhee J, Dayal R, Chaer R, Morrissey NJ, et al: Analysis of anatomic factors and age in patients undergoing carotid angioplasty and stenting. Ann Vasc Surg 19:798-804, 2005

4. Simmons CR, Tsao EC, Thompson JR: Angiographic approach to the difficult aortic arch: a new technique for transfemoral cerebral angiography in the aged. Am J Roentgenol Radium Ther Nucl Med 119:605-612, 1973

5. Vitek JJ, Roubin GS, Al-Mubarek N, New G, Iyer SS: Carotid artery stenting: technical considerations. AJNR Am J Neuroradiol 21:1736-1743, 2000

\section{Disclosures}

Dr. Siddiqui reports the following. Financial interests: Buffalo Technology Partners Inc., Cardinal, International Medical Distribution Partners, Neurotechnology Investors, Hotspur, Intratech Medical, StimSox, Valor Medical, Blockade Medical, Lazarus Effect, Pulsar Vascular, and Medina Medical. Consultant: Amnis Therapeutics Inc., Cerebrotech Medical Systems Inc., CereVasc LLC, Corindus Inc., Codman \& Shurtleff, Covidien Vascular Therapies, GuidePoint Global Consulting, Penumbra, Stryker,
Pulsar Vascular, MicroVention, Lazarus Effect, Blockade Medical, Reverse Medical, and W. L. Gore \& Associates. National steering committees: Penumbra-3D Separator Trial, CovidienSWIFT PRIME Trial, and MicroVention-FRED Trial. Speakers' bureau: Codman \& Shurtleff Inc. Advisory board: Codman \& Shurtleff, Covidien Neurovascular, ICAVL, and Medina Medical. Honoraria: Penumbra and Toshiba Medical Systems.

\section{Author Contributions}

Conception and design: all authors. Acquisition of data: all authors. Analysis and interpretation of data: all authors. Drafting the article: Rangel-Castilla. Critically revising the article: all authors. Reviewed submitted version of manuscript: all authors.

\section{Supplemental Information \\ Current Affiliations}

Dr. Rangel-Castilla: Department of Neurosurgery, Wayne State University, Detroit, MI.

\section{Correspondence}

Adnan H. Siddiqui, University at Buffalo Neurosurgery, 100 High St., Ste. B4, Buffalo, NY 14203. email: asiddiqui@ubns.com. 